\title{
Reemplazo valvular tricuspídeo: prótesis mecánica versus prótesis biológica. Trabajo observacional-descriptivo
}

\author{
Cecilia Muñoz*, Juan Contreras**, Pedro Becker, Rodrigo González, Ricardo Zalaquett. \\ Sección de Cirugía Cardíaca, Facultad de Medicina, Pontificia Universidad Católica de Chile. \\ ${ }^{*}$ Actualmente, en Hospital del Tórax, Santiago. \\ ${ }^{* *}$ Actualmente, en Hospital Regional de Temuco.
}

Financiamiento: Ninguno.

Antecedentes: El reemplazo protésico de la válvula tricúspide es un procedimiento infrecuente, con elevada mortalidad y morbilidad operatoria, independientemente de la etiología de la insuficiencia tricuspídea. Persiste aún una discusión respecto al tipo de prótesis a utilizar, mecánica o biológica.

Objetivo: Analizar nuestros resultados perioperatorios y alejados en el reemplazo valvular tricuspídeo, comparando ambos tipos de prótesis.

Método: Revisión de la Base de Datos de nuestro Servicio de Cirugía Cardiovascular para el periodo enero 1991 - diciembre 2017. Identificados los pacientes con reemplazo valvular tricuspídeo (RVT); se revisaron los protocolos operatorios y los ecocardiogramas. La supervivencia se certificó a través del Registro Civil e Identificación de Chile.

Resultados: Se identificaron 83 pacientes con RVT (76\% mujeres), los que representaron el $0,7 \%$ del total de las cirugías con circulación extracorpórea y el $2,1 \%$ de las cirugías valvulares para el periodo en estudio. La edad promedio fue $49 \pm 16,5$ años. Cuarenta y nueve casos (59\%) correspondieron a reoperaciones y otros 49 tuvieron un procedimiento asociado. En 40 pacientes (48\%) se utilizó una prótesis mecánica y en 43 (52\%) una biológica. La mortalidad operatoria global fue $9,6 \%$ ( 8 pacientes, 4 con una prótesis mecánica y 4 con una biológica). El seguimiento se completó en el $100 \%$, con un promedio de 7,1 años. Veintiocho pacientes fallecieron durante el seguimiento; la principal causa fue insuficiencia cardiaca. Así, la supervivencia a 5 años fue $70,3 \pm$ $5,3 \%$ y a 10 años $58 \pm 6,3 \%$, sin diferencia significativa entre ambos tipos de prótesis. Siete pacientes se reoperaron durante el seguimiento (5 casos con prótesis biológica y 2 mecánica).

Conclusión: El RVT continúa siendo un procedimiento infrecuente, con mayor incidencia en mujeres, en la quinta década de la vida. La mayoría de los pacientes presentaba comorbilidad y había tenido cirugía cardiovascular previa. La mitad de estos recibió una prótesis mecánica y la otra, biológica. No hubo diferencias significativas entre ambos tipos de prótesis en cuanto a mortalidad operatoria, supervivencia alejada o reoperación.

Palabras Claves. Válvula tricúspide; insuficiencia tricuspídea; reemplazo valvular tricuspídeo. 


\section{Tricuspide valve replacement: comparison of biological versus mechanical valve: a descriptive observational study}

Background: Tricuspid valve replacement (TVR) is an uncommon surgical procedure, associated with high mortality and morbidity. The use of biological or mechanical prostheses in TVR has advantages and disadvantages and, therefore, there persists a debate regarding the choice of one or other type of prostheses.

Aim: To analyze our operative and long-term surgical results, comparing both types of prosthetic valves.

Methods: The Data Base of the Cardiovascular Surgery Service was reviewed for the period between January 1991 and December 2017. 83 patients with TVR were identified, the operative notes and echocardiogram reports were analyzed. Survival was obtained from the Chilean Civil Identification Service.

Results: 83 patients (76\% women) had TVR. They represented $0.7 \%$ of the total cases operated on with extracorporeal circulation and $2.1 \%$ of all valve disease cases, for the study period. Mean age was $49 \pm 16.5$ years. 49 cases $(59 \%)$ were reoperations and another 49 had an associated procedure.
In 40 patients (48\%) a mechanical prosthesis was used and in $43(52 \%)$ a biological one was implanted. Operative mortality rate was $9.6 \%$ (8 patients, had a mechanical valve and the other 8 , a biological one). Follow-up was $100 \%$ completed, with an average of 7.1 years. 28 patients died during follow-up; the main cause of death was heart failure. Five-year survival rate was $70.3 \pm 5.3 \%$ and at 10 years it was $58 \pm 6.3 \%$, without significant difference the type of prostheses. Seven patients were re-operated during follow-up ( 5 cases corresponded to a biological prostheses and 2 to a mechanical one).

Conclusion: TVR is still an infrequent surgical procedure, more commonly performed in women, on the fifth decade of life. Most patients presented comorbidities and had a previous cardiovascular surgical operation. Half of them received a mechanical prosthesis and half a biological one. There was no significant difference between both types of prostheses related to surgical mortality, long-term survival or reoperation.

Keywords: Tricuspid valve. Tricuspid valve replacement. Tricuspid insufficiency. 


\section{Introducción:}

La Insuficiencia Tricuspídea (IT) "leve" es un hallazgo frecuente en pacientes en quienes se efectúa un estudio ecocardiográfico, sin consecuencias fisiopatológicas para ellos. Por otra parte, la IT significativa es, en la mayoría de los casos (aproximadamente 80\%) "funcional", por dilatación del anillo tricuspídeo y tracción de los velos, por remodelamiento del ventrículo derecho y esto, a su vez, por sobrecarga de presión y/o volumen, secundarias a patología cardíaca izquierda. La IT "primaria” o "valvular", aunque más infrecuente, es casi siempre significativa o importante, siendo las etiologías más frecuentes la enfermedad reumática, la degeneración mixomatosa, la enfermedad de Ebstein, carcinoide, actínica, la presencia de electrodos de marcapaso o de desfibrilación, entre otras ${ }^{1,2}$.

Si bien la indicación de anuloplastía tricuspídea con anillo protésico concomitante está en la actualidad bien definida para pacientes con insuficiencia tricuspídea funcional, que se operan primariamente por patología valvular izquierda, no lo es así para la IT primaria o para los casos de reoperación por IT funcional severa, asociada a distintos grados de insuficiencia cardiaca derecha, en pacientes previamente operados por patología valvular aórtica o mitral, en los que el reemplazo tricuspídeo debe ser también considerado ${ }^{3-6}$. En estos casos hay, además, controversia respecto del tipo de prótesis a utilizar. Las Guías Clínicas AHA/ACC 2014 recomiendan individualizar esta elección en la forma habitual, considerando los riesgos de trombosis y anticoagulación de las prótesis mecánicas y la durabilidad limitada de las prótesis biológicas ${ }^{1}$.

En este trabajo, observacional y descriptivo, analizamos nuestros resultados perioperatorios y alejados en pacientes sometidos a Reemplazo Valvular Tricuspídeo (RVT) en el Servicio de Cirugía Cardiovascular, comparando ambos tipos de prótesis en cuanto a sexo y edad, etiología y comorbilidad, mortalidad operatoria y alejada y reoperación.

\section{Método:}

Para la identificación de los pacientes se revisó la base de datos del Servicio de Cirugía Cardiovascular, para el período comprendido entre enero 1991 y diciembre 2017. Identificados los pacientes con RVT, se revisaron los protocolos operatorios y los informes ecocardiográficos disponibles. La supervivencia alejada se certificó a través del Registro Civil e Identificación de Chile. Se incluyeron todos los pacientes operados en forma consecutiva en el período mencionado.
Se determinaron variables demográficas e incidencia de RVT, comorbilidad, cirugía previa, etiología y procedimientos quirúrgicos asociados para todo el grupo y luego se determinó el tipo de prótesis utilizado, comparando entre éstas, sexo y edad de los pacientes, mortalidad operatoria y supervivencia y reoperación en el seguimiento alejado.

Análisis estadístico. Las variables categóricas se expresan en porcentaje y las variables numéricas en promedio y margen. La significación estadística se evaluó mediante prueba $t$ de Student para muestras no pareadas en el caso de variables continuas y prueba de Chi cuadrado para comparaciones de frecuencias. Las curvas de supervivencias se construyeron por medio del método de Kaplan-Meier. Un valor alfa de 5\% fue definido a priori para determinar diferencias estadísticas significativas. Se usó el paquete estadístico SPSS en su versión 17.0 (SPSS Inc., Chicago, USA).

Por tratarse de un estudio de revisión, sin interacción con los pacientes, con anonimización de información, y de acuerdo con el Comité Ético Científico institucional, no se requirió de consentimiento informado.

\section{Resultados:}

Pacientes. Se identificaron 83 pacientes con RVT para el periodo en estudio, 63 (76\%) eran mujeres. La edad promedio fue 49 $\pm 16,5$ años (margen: 16 a 82 años). En este periodo (enero 1991 a diciembre 2017) se efectuaron un total de 11.262 cirugías cardíacas con circulación extracorpórea, en pacientes mayores de 15 años. De estas, 3.901 (35\%) correspondieron a cirugías valvulares y, de estas, 695 (18\%) tuvieron un procedimiento quirúrgico sobre la válvula tricúspide. A su vez, de estos últimos $613(88 \%)$ correspondieron a anuloplastías tricuspídeas y $83(12 \%)$ a reemplazo tricuspídeo. En todos estos últimos, la indicación operatoria fue una insuficiencia tricuspídea ecocardiográficamente severa, asociada a síntomas y signos de insuficiencia cardíaca derecha, de intensidad variable.

En otros términos, estos 83 casos de RVT correspondieron al 0,7\% del total de las cirugías con circulación extracorpórea y al 2,1\% del total de las cirugías valvulares en el periodo en estudio.

Comorbilidad. Cuarenta y cinco pacientes (54\%) estaban en arritmia completa por fibrilación auricular, 21\% eran hipertensos y $16 \%$ tenía antecedentes de un accidente cerebrovascular. (Tabla 1). 


\begin{tabular}{lcc}
$\begin{array}{l}\text { Tabla 1: Morbilidad preoperatoria asociada al reemplazo } \\
\text { tricuspídeo }\end{array}$ & n \\
\hline Morbilidad asociada & 45 & 54,2 \\
\hline Fibrilación Auricular permanente & 17 & 20,5 \\
\hline Hipertensión Arterial & 13 & 15,7 \\
\hline Accidente Cerebro Vascular & 13 & 15,7 \\
\hline Hipertensión pulmonar & 8 & 9,6 \\
\hline Diabetes Mellitus & 6 & 7,2 \\
\hline Cardiopatía coronaria & 5 & 6,0
\end{tabular}

Cirugía previa. Cuarenta y nueve pacientes (59\%) habían tenido una cirugía cardiovascular previa; de estos, 8 habían tenido 2 cirugías previas y uno, ${ }^{3}$. Las cirugías más frecuentes fueron el reemplazo mitral $(45 \%)$ y la comisurotomía mitral (33\%). Doce pacientes (25\%) habían tenido una anuloplastía tricuspídea previa. (Tabla 2).

Tabla 2: Procedimientos quirurgos realizados previamente al reemplazo tricuspídeo.

\begin{tabular}{lc}
\hline Procedimiento & $\mathrm{n}(\%)$ \\
\hline Reemplazo valvular mitral & $22(45)$ \\
\hline Comisurotomía mitral & $16(33)$ \\
\hline Anuloplastía tricúspide & $12(24,5)$ \\
\hline De Vega & 7 \\
Con anillo & 5 \\
\hline Reparación patología congénita & $7(14)$ \\
\hline Reemplazo valvular aórtico & $6(12)$ \\
\hline Reparación malformación de Ebstein & $2(4)$ \\
\hline Resección Mixoma & $1(2)$
\end{tabular}

Etiología. La secuela reumática, la dilatación del anillo tricuspídeo secundaria a valvulopatía izquierda y la Enfermedad de Ebstein, fueron las etiologías más frecuentes (Tabla 3).

Procedimientos asociados. Cuarenta y nueve pacientes (59\%) tuvieron al menos un procedimiento cardioquirúrgico asociado. Los más frecuentes fueron el reemplazo mitral en 27 casos, y el implante de electrodos de marcapaso epicárdico, en 31 (Tabla 4).

Tipo de prótesis. En 40 pacientes (48\%) se utilizó una
Tabla 3: Etiología de la valvulopatía tricuspídea.

\begin{tabular}{lcc}
\hline Etiología & $\mathrm{n}$ & $\%$ \\
\hline Reumática & 20 & 24 \\
\hline Dilatación anillo $2^{\circ}$ patología izquierda & 16 & 19 \\
\hline Malformación de Ebstein & 14 & 17 \\
\hline Endocarditis Infecciosa & 8 & 9,6 \\
\hline Congénita no Ebstein & 6 & 7,2 \\
\hline Carcinoide & 5 & 6 \\
\hline Disfunción prótesis tricúspide & 4 & 4,8 \\
\hline Otras & 10 & 12 \\
\hline Total & 83 & 100 \\
\hline
\end{tabular}

Tabla 4: Procedimientos quirúrgicos asociados al reemplazo valvular tricúspideo, en 49 pacientes.

\begin{tabular}{lc} 
Procedimiento & $\mathrm{n}$ \\
\hline Reemplazo Valvular Mitral & 27 \\
\hline Implante Electrodo epicárdico & 31 \\
\hline Cierre defectos (CIA/CIV) & 6 \\
\hline Reemplazo valvular aórtico & 7 \\
\hline Reemplazo valvular pulmonar & 4 \\
\hline Revascularización miocárdica & 3 \\
\hline Plastia válvula mitral & 3 \\
\hline Pericardiectomía & 2 \\
\hline Plastia válvula aórtica & 1 \\
\hline Resección mixoma & 1 \\
\hline Cirugía de arritmias (ablación) & 3 \\
\hline TOTAL PROCEDIMIENTOS & 88 \\
\hline
\end{tabular}

prótesis mecánica (St. Jude en 35, Starr-Edwards en 3 y Carbomedic en 2) y en 43 pacientes (52\%) una prótesis biológica (Carpentier-Edwards en 41 y St. Jude Epic en 2). En la mayoría de los pacientes con enfermedad reumática se utilizó una prótesis mecánica; por el contrario, en la mayoría de los pacientes con Enfermedad de Ebstein el RVT se efectuó con una prótesis biológica (Tabla 5). No hubo diferencias estadísticamente significativas en el tipo de prótesis utilizada en cuanto a sexo, pero sí en relación con la edad de los pacientes, puesto 


\begin{tabular}{|c|c|c|}
\hline Etiología & Biológica & Mecánica \\
\hline Reumática & 3 & 17 \\
\hline Dilatación anillo $2^{\circ}$ patología izquierda & 6 & 10 \\
\hline Malformación de Ebstein & 12 & 2 \\
\hline Endocarditis Infecciosa & 6 & 2 \\
\hline Congénita no Ebstein & 4 & 2 \\
\hline Carcinoide & 1 & 4 \\
\hline Disfunción prótesis tricúspide & 4 & 0 \\
\hline Otras & 7 & 3 \\
\hline Total & 43 & 40 \\
\hline
\end{tabular}

Tabla 6: Sexo y edad de Reemplazo valvular tricúspideo según tipo de prótesis utilizada.

\begin{tabular}{|l|c|c|c|}
\hline & Biológicas & Mecánicas & $p$ \\
\hline Sexo & & & 0,206 \\
\hline Femenino & $30(70 \%)$ & $33(82,5 \%)$ & \\
\hline Masculino & $13(30 \%)$ & $7(17,5 \%)$ & \\
\hline Edad & $44,1 \pm 17,4$ & $53,4 \pm 14,3$ & 0,01 \\
\hline
\end{tabular}

que en los pacientes más jóvenes las prótesis biológicas se utilizaron más frecuentemente (Tabla 6).

El tamaño protésico promedio de las prótesis de St. Jude fue $30,2 \pm 2,1$ y la mediana 31 ; para las prótesis de Carpentier-Edwards el tamaño promedio fue $30,1 \pm 1,8$ y la mediana 31 .

Mortalidad operatoria. La mortalidad operatoria (30 días) fue $9,6 \%$ (8 pacientes). La mitad recibió una prótesis mecánica y la otra una prótesis biológica (NS).

Seguimiento alejado. El seguimiento se cerró en diciembre 2018 y se completó en el 100\% de los casos, con un promedio de 7,1 años ( 1 a 24 años). Veintiocho pacientes (37\%) fallecieron durante el periodo de seguimiento; 16 habían tenido un RVT con prótesis mecánica y 12 con prótesis biológica. Las causas de muerte fueron insuficiencia cardiaca en 16 pacientes, infecciosa en 5, cáncer en 3, hemorrágica en 3 e insuficiencia renal en 1 paciente. Así, la supervivencia a 5 años fue $70,3 \% \pm 5,3 \%$ y a 10 años $58,0 \% \pm 6,3 \%$ (Figura 1). No hubo diferencia significativa en la supervivencia alejada entre ambos tipos de

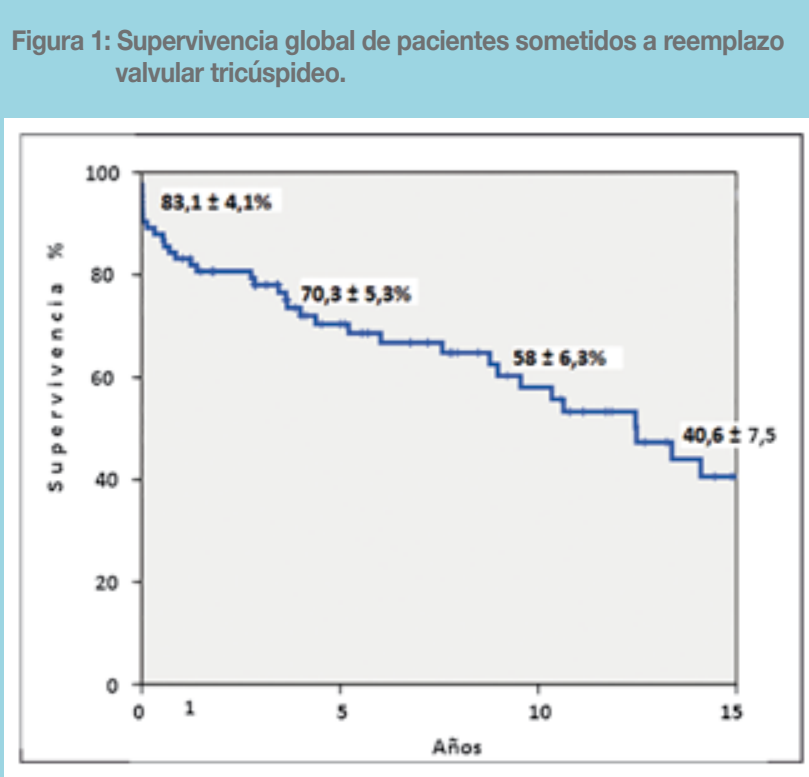

Figura 2: Supervivencia según tipo de prótesis utilizada en pacientes sometidos a reemplazo valvular tricúspideo.

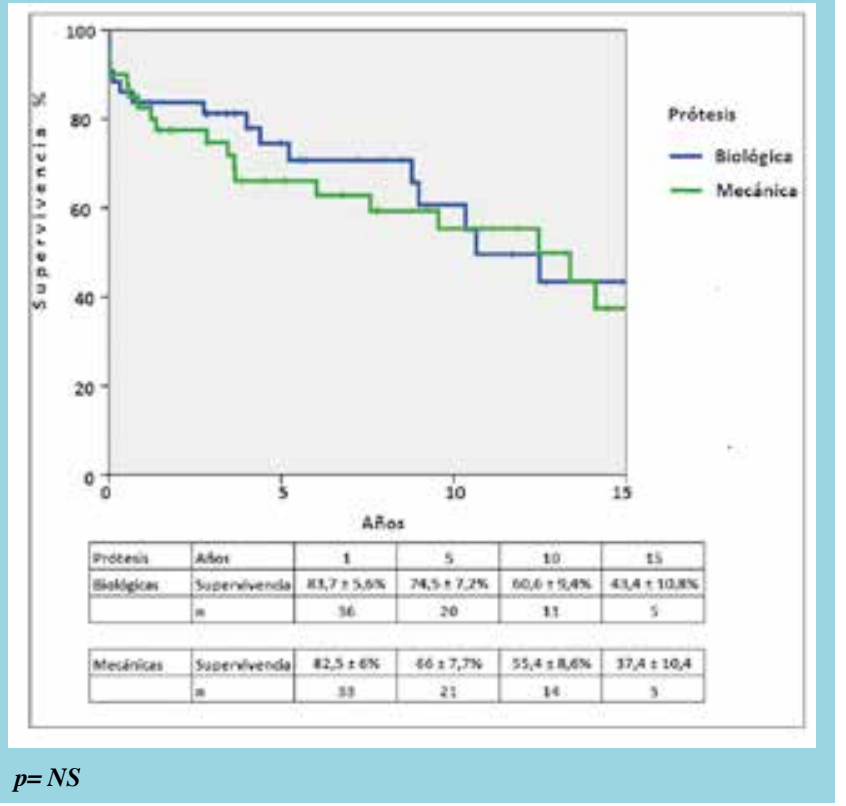

prótesis (Figura 2).

Reoperación. Siete pacientes $(9,3 \%)$ se reoperaron en nuestra institución durante el seguimiento. Cinco casos correspondieron a prótesis biológica y 2 a prótesis mecánica. La indicación fue una disfunción estructural en los 5 casos de prótesis biológica; en los 2 casos de prótesis mecánica reoperados, la causa fue una endocarditis en uno y una trombosis en el otro. La supervivencia libre de reoperación, fue de 20,4 años (IC95\%: 17,8 - 
Figura 3: Supervivencia libre de reoperación de pacientes sometidos a reemplazo valvular tricuspídeo.

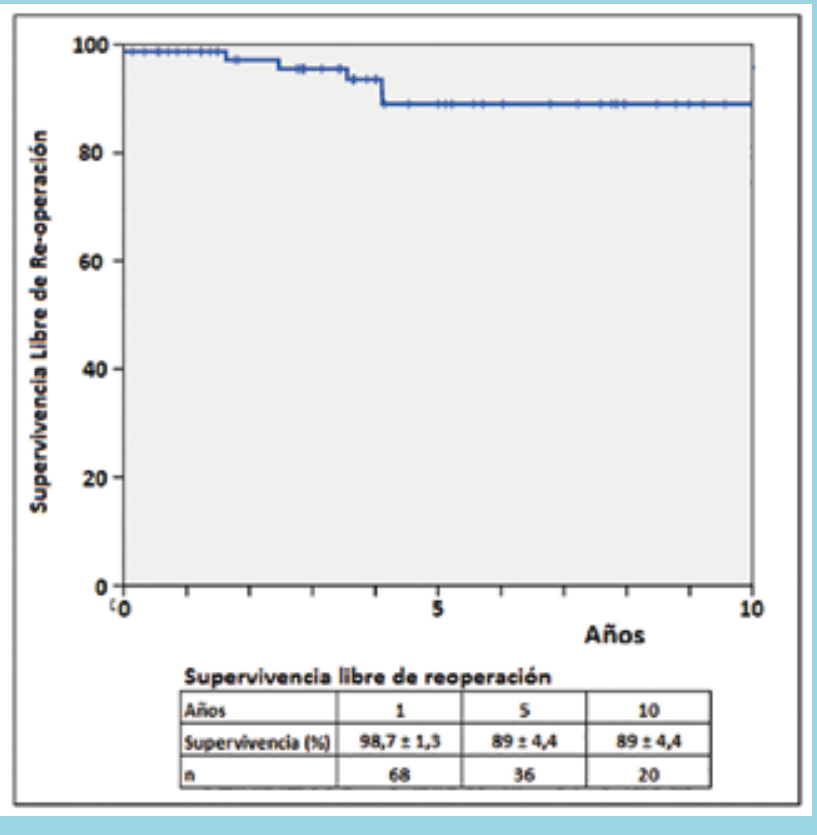

$23,0)$, sin diferencia estadísticamente significativa entre ambos tipos de prótesis (Figura 3).

\section{Discusión.}

La cirugía sobre la válvula tricúspide siempre ha sido un gran desafío.

Se efectúa infrecuentemente por la mayoría de los cirujanos cardiacos, siendo mucho más frecuente la anuloplastía tricuspídea ${ }^{1-6}$. Solo ocasionalmente una IT muy severa, ya sea funcional u orgánica, es tratada con un RVT. Por lo mismo, el tipo de prótesis a utilizar ha sido y es motivo de controversia, originada en la menor durabilidad de las prótesis biológicas versus las complicaciones relacionadas a la anticoagulación requerida por las prótesis mecánicas 1,3-5,7-9.

En general, el RVT representa, en la mayoría de las series, menos del $2 \%$ de toda la cirugía valvular; en nuestro caso, en el período analizado el RVT representó el $2,1 \%$ de toda la cirugía valvular y el $0,7 \%$ de toda la cirugía con circulación extracorpórea. Al igual que en nuestra experiencia, la mayoría de los pacientes son adultos jóvenes, de edad promedio alrededor de 50 años y la mayoría mujeres; en nuestra experiencia estas representaron el $76 \%$ de los $\operatorname{casos}^{9}$.

Un porcentaje importante de los pacientes que requieren RVT presentan, como en nuestra serie, comorbilidad y
Figura 4: Supervivencia libre de reoperación según tipo de prótesis.

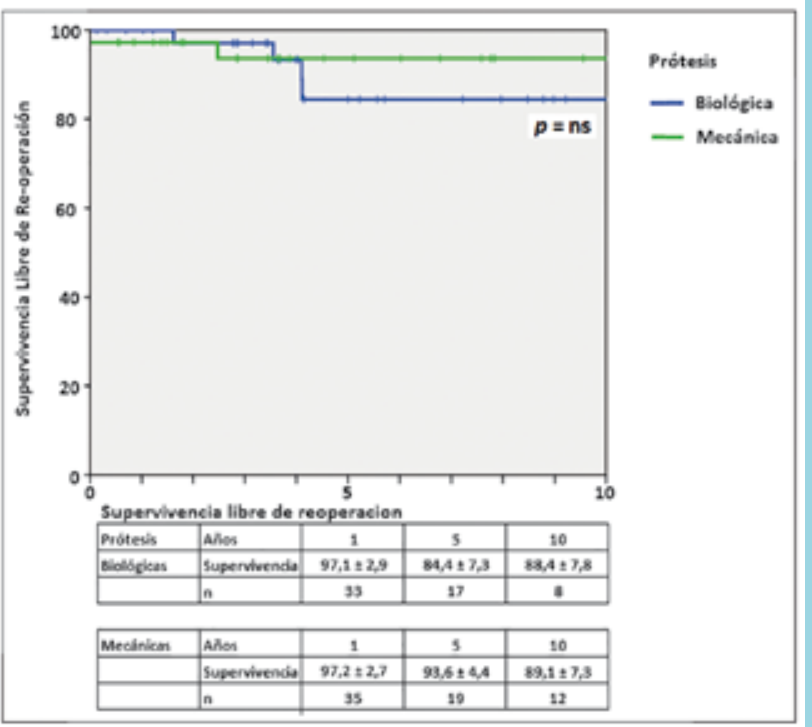

en la mayoría de los casos, 59\% en nuestra experiencia, han tenido una cirugía previa, lo que incrementa el riesgo operatorio ${ }^{7-9}$. Así, la mortalidad operatoria, reportada en distintas series, es entre $12 \%$ a $26 \%$, comparándose favorablemente nuestra mortalidad operatoria de 9,6\%.7-11. Igualmente, como en otras series, no hubo diferencias significativas entre ambos tipos de prótesis, ya que de los 8 pacientes que fallecieron en el periodo perioperatorio, 4 recibieron una prótesis biológica y los otros 4 una prótesis mecánica ${ }^{7-11}$. Una causa importante de mortalidad operatoria es la reoperación valvular tricuspídea ${ }^{10,11}$, la que fluctúa entre $10 \%$ y $25 \%$ en las distintas series (bastante menor a nuestra experiencia) Ello ha significado que, ante cualquier grado de insuficiencia tricuspídea, es frecuente efectuar una anuloplastía tricuspídea complementaria. En los casos de cirugía valvular izquierda primaria, ante cualquier grado de IT con dilatación del anillo se procede a la anuplastía de la válvula. Así, de acuerdo con las últimas guías clínicas AHA/ACC, ésta debe efectuarse si ecocardiográficamente el diámetro diastólico del anillo tricuspídeo es $\geq 40 \mathrm{~mm}$ o $\geq 70 \mathrm{~mm}$ en una medición intraoperatoria directa. (Es necesario consignar, sí, que esta recomendación se basa en un solo estudio de Dreyfus et al. publicado en 2005) $)^{1,12}$.

Por otra parte, la mayoría de nuestros pacientes requirieron, como en casi todas las series publicadas, un procedimiento cardioquirúrgico asociado, siendo los más frecuentes el reemplazo mitral y el implante de electro- 
dos de marcapaso epicárdico. Esto último, en la mayoría de los casos, como procedimiento asociado profiláctico ante un eventual requerimiento posterior de marcapaso definitivo, cuyo implante intracavitario sería imposible en caso de prótesis mecánica y desaconsejable en caso de prótesis biológica ${ }^{7-11}$.

Aunque en las guías clínicas AHA/ACC 2014 se recomienda que la elección entre un tipo de prótesis u otra debe individualizarse de acuerdo con la "negociación" habitual entre las complicaciones trombo hemorrágicas de las prótesis mecánicas versus la menor durabilidad de la prótesis biológica. Los metaanálisis no han demostrado diferencias significativas en cuanto a sobrevida entre ambos tipos de prótesis en el RVT. La tendencia reciente en la mayoría de los centros, pero no en todos, era preferir las prótesis biológicas por sobre las mecánicas basado en el hecho de que el supuesto menor estrés al que son sometidas las prótesis de tejido en las cavidades derechas contribuiría a su mayor durabilidad, inclusos en pacientes jóvenes. Si bien esto es difícil de probar, es un hecho empírico y en nuestra serie la sobrevida libre de reoperación en pacientes con prótesis biológica no fue inferior a la de aquellos con prótesis mecánicas, aun siendo más jóvenes. Por otra parte, las mismas razones se han esgrimido para un supuesto mayor riesgo de trombosis de las prótesis mecánicas en posición tricuspídea, dado los flujos más lentos trans protésicos $1,3,7-11$. Si bien esto último podría ser cierto con los modelos antiguos de prótesis mecánicas, no lo ha sido con la prótesis de St. Jude con tratamiento anticoagulante moderno, basado en INR y asociado a aspirina en dosis. Además, es conveniente hacer todo el esfuerzo posible por utilizar prótesis de gran tamaño, como ocurrió en esta experiencia. Ello contribuye a no tener un gradiente transvalvular elevado que puede originar no solo ectasia venosa, sino, además, un aumento de la presión venosa central lo que puede ser causa de una persistencia de insuficiencia cardíaca derecha ${ }^{8,9,13,14}$.

En nuestra serie, prácticamente la mitad de los pacientes recibió una prótesis biológica y la otra, una prótesis mecánica de Carpentier-Edwards en el primer caso, y de Saint Jude, en el segundo. No hubo diferencias en el tipo de prótesis utilizada en cuanto a sexo, pero sí en cuanto a la edad de los pacientes, recibiendo los más jóvenes (promedio de edad $44 \pm 17,4$ años) con mayor frecuencia una prótesis biológica. Ello puede explicarse por el número de pacientes con enfermedad de Ebstein y a preferencias individuales de los cirujanos en estos casos.
La supervivencia alejada del RVT ha sido, en general, poco satisfactoria, reportándose cifras entre $33 \%$ a $68 \%$, a 10 años, sin diferencias significativas entre ambos tipos de prótesis, lo que corrobora nuestro propio estudio, en el que la mortalidad a 10 años, independientemente del tipo de prótesis, fue $58,0 \pm 6,3 \%$. Igualmente, la principal causa de muerte es insuficiencia cardíaca. Una explicación para esto es que los pacientes con IT severa han sido históricamente operados muy tardíamente en el curso natural de la enfermedad, una vez que estos se han hecho sintomáticos, con signos de falla cardíaca derecha e hipertensión pulmonar ${ }^{3,7-11}$.

En el seguimiento alejado 7 pacientes $(9,3 \%)$ requirieron una reoperación tricuspídea, cifra similar a los pocos estudios que reportan este evento ${ }^{3,7-11}$. Esto se tradujo en una probabilidad promedio de estar vivo libre de reoperación de 20,4 años. Si bien 5 de estos 7 casos correspondieron a prótesis biológicas, esta diferencia no alcanzó significancia estadística. Esta baja incidencia de reoperación, menor a la que se podría esperar puede explicarse, al menos en parte, por la elevada mortalidad durante el seguimiento ya que al término de este, solo el 53\% de los pacientes estaban vivos y, por lo tanto, expuestos a necesitar una reoperación. Si bien solo se registró un paciente con prótesis mecánica que requirió reoperación por trombosis de prótesis, y no se identificó ningún paciente con prótesis tratado con trombolisis. Por otra parte, la presencia de una prótesis biológica no es sinónimo de ausencia de tratamiento anticoagulante, ya que un porcentaje importante de los pacientes portadores de estas requieren anticoagulación por otras razones, como fibrilación auricular, trombos auriculares o presencia de prótesis mecánicas izquierdas. Por lo mismo, la incidencia de complicaciones tromboembólicas o hemorrágicas es similar en ambos tipos de prótesis, en diferentes estudios ${ }^{7-11}$.

En conclusión, en nuestra experiencia, al igual que en la mayoría de las series, el RVT es infrecuente, con mayor incidencia en mujeres, en la quinta década de vida. La mayoría de los pacientes presentan comorbilidad tienen cirugía cardiovascular previa, a pesar de lo cual la mortalidad operatoria fue menor a la reportada. La mitad de estos recibió una prótesis mecánica y la otra una biológica y no hubo diferencias significativas entre ambos tipos en cuanto a mortalidad operatoria, supervivencia alejada o reoperación. De aquí que, en nuestra opinión, la recomendación de las Guías Clínicas AHA/ACC es válida, en cuanto a seleccionar el tipo de prótesis considerando los riesgos de trombosis y anticoagulación. 
Limitaciones del estudio. Como en prácticamente todas las series publicadas, nuestro estudio corresponde a un análisis retrospectivo $\mathrm{y}$, por lo tanto, adolece de todas las limitaciones propias de este tipo de estudio, incluyendo la heterogeneidad de los pacientes. Sin embargo, representa la realidad de un centro cardioquirúrgico nacional en los últimos 25 años y sus resultados son concordantes con los trabajos relacionados más recientemente publicados. Del mismo modo, dadas las características propias de la enfermedad y su tratamiento quirúrgico, un estudio prospectivo, randomizado y ciego resulta prácticamente imposible.

Una limitante particular, es que este estudio solo pudo identificar a los pacientes que fueron reoperados durante el seguimiento en nuestra institución.

\section{Agradecimientos:}

Agradecemos a los Dres. Cristian Baeza, Manuel Irarrázaval, Gustavo Maturana, Sergio Morán, Miguel Navarro y Gabriel Olivares, quienes operaron a algunos de los pacientes incluidos en este estudio.

\section{Referencias}

1. NISHIMURA RA, OTTO CM, BONOW RO, CARABELLO BA, ERWIN JP, GUYTON RA, et al. 2014 AHA/ACC Guidelines for the management of patients with valvular heart disease. Circulation 2014;129:e521-e643.

2. NATH J, FOSTER E, HEIDENREICH PA. Impact of tricuspide regurgitation on long-term survival. J Am Coll Cardiol 2004;43:405-9.

3. VASSILEVA CM, SHABOSKY J, BOLEY T, et al. Tricuspide valve surgery: the past 10 years from the Nationwide Inpatient Sample (NIS) database. J Thorac Cardiovasc Surg 2012;143:1043-9.

4. ROGERS JH, BOLLING SF. The tricuspide valve: current perspective and evolving management of tricuspide regurgitation. Circulation 2009;119:2718-25.

5. CURI P, MORÁN S, CÓRDOVA S, ZALAQUETT R, CHAMORRO G, IRARRAZAVAL M, BECKER P, ARRETZ C. Tratamiento quirúrgico de la insuficiencia tricúspide funcional: Análisis de dos técnicas de valvuloplastía. Rev Chil Cardiol 2004;23:353-362.

6. BAHAMONDES JC, GODOY R, SILVA A, DÍAZ A, PEÑA M. Reparación de la insuficiencia tricuspídea: resultados a largo plazo en 114 pacientes. Rev Chil Cardiol 2018;37:85-92.

7. KUNADIAN B, VIJAYALAKSHMI K, BALASUBRAMANIAN $S$, et al. Should the tricuspide valve be replaced with a mechanical or biological valve? Interact Cardiovasc Thorac Surg 2007;6:551-7.
8. ALTAANI HA, JABER S. Tricuspid valve replacement, mechanical vs. biological valve, which is better? Int Cardiovasc Res j 2013;7:71-4.

9. SONGUR CM, SIMSEK E, OZEN A, et al. Long term results comparing mechanical and biological protheses in the tricuspide position. Which valve types are better - mechanical or biological prostheses? Heart, Lung and Circulation 2014;1615:1-4.

10. RIZZOLI G, VENDRAMIN I, NESSERIS G, et al. Biological or mechanical prostheses in tricuspide position? A meta-analysis of intra-institutional results. Ann Thorac Surg 2004;77:1607-14.

11. FILSOUFI F, ANYANWU AC, SALZBERG SP, et al. Longterm outcomes of tricuspid valve replacement in the current era. Ann Thorac Surg 2005;80:845-50.

12. DREYFUS GD, CORBI PJ, CHAN KM, et al. Secondary tricuspid regurgitation or dilatation: Which should be the criteria for surgical repair? Ann Thorac Surg 2005;79:127-32.

13. SING AK, CHRISTIAN FD, WILLIAMS DO, et al. Follow-up assessment of the St. Jude Medical prosthetic valve in the tricuspid position: clinical and hemodynamic results. Ann Thorac Surg 1984;37:324-7.

14. SUNG K, PARK PW, PARK K-H, et al. Is tricuspid valve replacement a catastrophic operation? European Journal of Cardio-thoracic Surgery 2009;36:825-9. 